\title{
Scalable Cloud Platform for Smart Home Devices Automation
}

\author{
SHRIMAN K.ARUN \\ \{shrirocksfire@gmail.com\} \\ Class 12, D.A.V BOYS SR. SEC. SCHOOL(CBSE), GOPALAPURAM,CHENNAI-14
}

\begin{abstract}
Empathize the user needs for home device automation is a creative design thinking to facilate man kind. The prevalence of home automation has been increased due to inter-linking and shadowing of human activities by controled machine activities. Use of machines and its control by using information technologies to improve automation is a next step beyond mechanization. Control home machinaries, electronic devices, fire alarm systems, water tank management systems, and security surveillance camera can be automate to reduce manual labour, elecrticty and ease of accesing from remote systems to make human living style as easy and secure. This paper, cloud based support for home devices automation bolster the elders, pet animal care takers and disabled persons activity by remote automation though Smartphone and tablet connectivity. Focus of this project is to develop a system which makes integration of the different home automation components seamlessly irrespective of manufacturer or where the components are coming from and accessing by remote by storing in cloud.
\end{abstract}

Keywords: Home automation, IoT, affordability and simplicity.

\section{Introduction}

Popularization of home automation needs the following hardware and software

Host Controller: Controllers are the "brains" of an intelligent home automation system. They allow you to integrate and control security, heating and air, lighting, entertainment and other home systems with simple convenience. Home Controls provides system hubs and smart home controllers from the biggest names in Home Automation and Security

RTOS F/W: AnRTOS is a piece of software designed to efficiently manage the time of a central processing unit (CPU)for microcontrollers and small microprocessors. RTOS-a real-time operating system kernel is used as micro controller platforms, AliOS Things -AliOS (formerly Yun OS and Aliyun OS) is a Linux distribution developed by Alibaba Cloud, and Arm MBED OS-Arm Mbed OS is a free, open-source embedded operating system designed specifically for the "things" in the Internet of Things.

Transducer: A transducer converts a signal to a usable form Transducers are deployed for automation, control and measurements, as well as the electrical signals converts physical quantities such as energy, torque, motion and light ).

Security: Security components are used for secure the data communication ie. Encrypt the data while it is being communicating between sensor to backend devices / applications

Cloud Backend:-There are numerous options available for cloud based backend, foremost beings are Amazon, Azure and Alibaba Cloud. Cloud Backend is where the web, data storage and analytic services are installed.

-Other Miscellaneous add-on features

Other than above mentioned different services, data visualization and data access by users play an important role in success of the solution as well. So there are number of solutions which can be used for data visualization. Since smart devices are very handy these days, users wish to access the data onsmart phones / handheld devices. Among them, most popular one are android based or iOS based.Components Requirement for making smart home solutions are the following and is shown in Figure 1:-- 


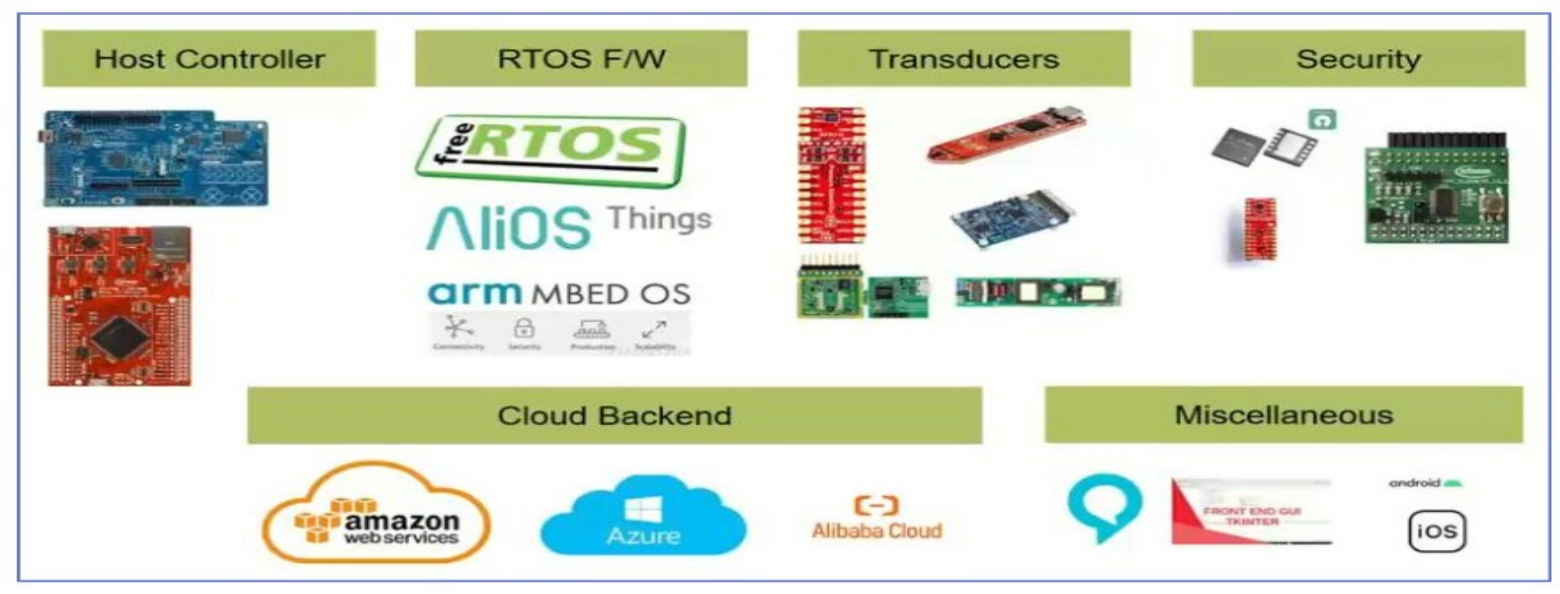

Fig.1.Components of Smart Home

\section{Significance of home automation in the modern era:}

Smart homes connect all electrical, electronics, camera and sensors and controlled by IoT protocols to facilitate users to empathises the user needs. It is very much useful for elderly persons, Pet care takers and checking the water overflow conditions [1]. More than 50 million smart speakers have been installed in American households [2]. Sensors senses the data from the home apparatus such as AC, DC Light and water tank sensor data [3][8].Sensors accurately sense the real-time data disaster management data such as fire detection alarm with high accuracy [4][7]. Cloud computing provide a variety of computing services in various levels and provide minimal interaction [5][6] to manage smart home data.

\section{ProblemDefinition \& Process Mapping}

\section{Phase 1:}

The field of Automation has well advanced in Industries, as majority of automobile industry plants as well as Oil $\&$ Gas plants have automated assembly lines. But automation has not yet penetrated in the homes as expected, especially in Middle East and India. If automation were to be used in homes than everyday life would be much easier. Simple example of use of automation in home can be seen in lighting where individual lights can be grouped into rooms and gives the user the ability to switch room on/off or change the colour or brightness of all the lights to match the user's moods or their activities. It is easy to control no matter how many lights are present. Similarly, there are many other things like home theatre, ac, washing machine fire alarm etc. in home which can be automated and integrated to a centralized dashboard to give a holistic view and management of the same. Therefore, the focus of this project is to develop a system which makes integration of the different home automation components seamlessly irrespective of manufacturer or where the components are coming from. This is the reason the project is classified as "Smart Home System" rather than Home Automation System.

Project Target-Smart home in a scaled down manner-

Highlighting interconnection between different smart devices-Seamless data transfer enhancing the user experience Key Highlights-Micro services based decentralized software-AWS Free RTOS based Firmware Application-Seamless Integration of different smart products Demonstration in Local Mode-Smart Light -Alexa Powered-Sensor Hub -Local Sensor Node-Smart AC -Simulation of an AC controlled by Alexa \& Sensor Hub Final Project Outcome (AWS Based) \& Demonstration-Fully Software Architecture-Full System Block DiagramSimulated light and AC-Sensor Hub uploading Pressure \& Temperature-Alex Control

Problem Definition-this project will not only make the life easier for people but also the system scalable i.e. developing a system which would be capable of integrating different home automation components irrespective of it coming from different manufactures and thus, making a vendor independent system. Following is the scope of the project-Identification of business requirement-Smart Lights,Security Camera,Smart Home Theatre,Smart AC,Smart Washing,MarchinAlexa Voice Service for Voice Command . 
-Subsystem not integrating:-When systems comes from different vendors, there will be always an issue in integration like security camera not connecting, entertainment units dropping out, lights are not responding etc. The issue could be that subsystems are not integrating as they should. So the focus of this solution is to design system with which easy integration can be done.

-One of the most common problem with smart home is that the system installed is without sufficient features and functionality. The idea is to design solution to integratedifferent home automation components into single integrated dashboard to better manage the all the devices, offering a Systemthat will have a centralized dashboard -People with special need always has issue managing different equipment at home and thus, this solution will have a system which can operate with voice command, making life easier especiallyforpeople with special needs.

Process Mapping, Sensor Hub, Smart Light, and Air Conditioner. A good point to mention here that everything is controlled by Alexa. A XMC800 which isInfineon Controller is being used as the main board. Hardware security solution for sensor hub and air conditioner are also being used here. However, hardware security is not used for smart light. The hardware security module TRUST X/M alsocomes from Infineon. To demonstrate sensors, Infineon DPS310 which is pressure and temperature sensor is being used. For light control, InfineonXMC controller is used, and motor control is powered byInfineonIMM102Tcontroller as shown inFigure 2,3 and 4.

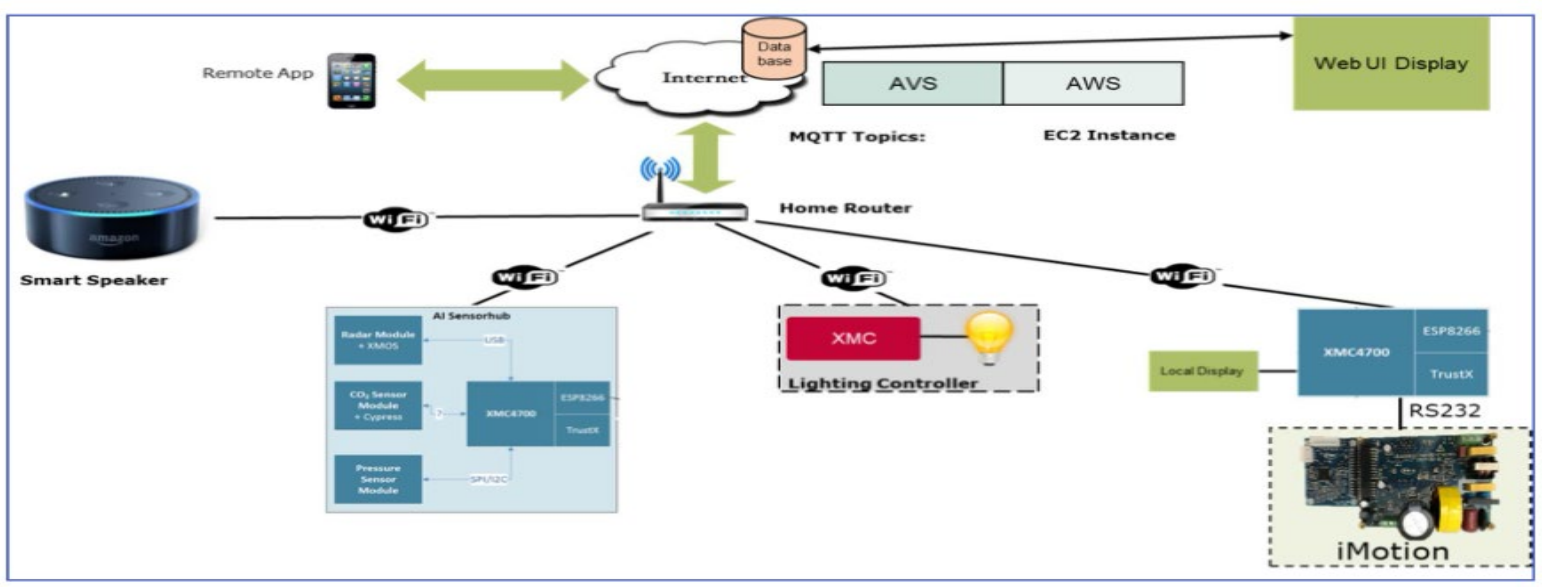

Fig.2.High Level View

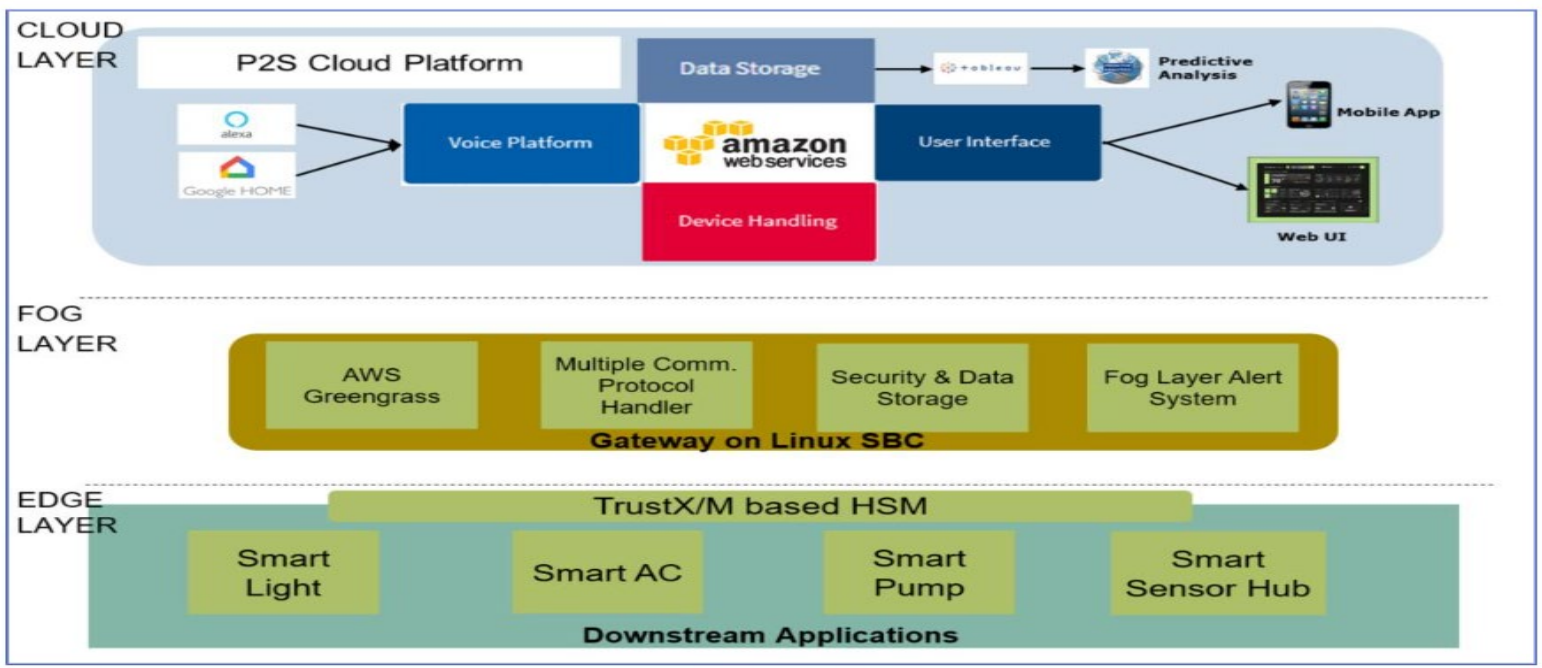

Fig.3.Abstract Architecture 


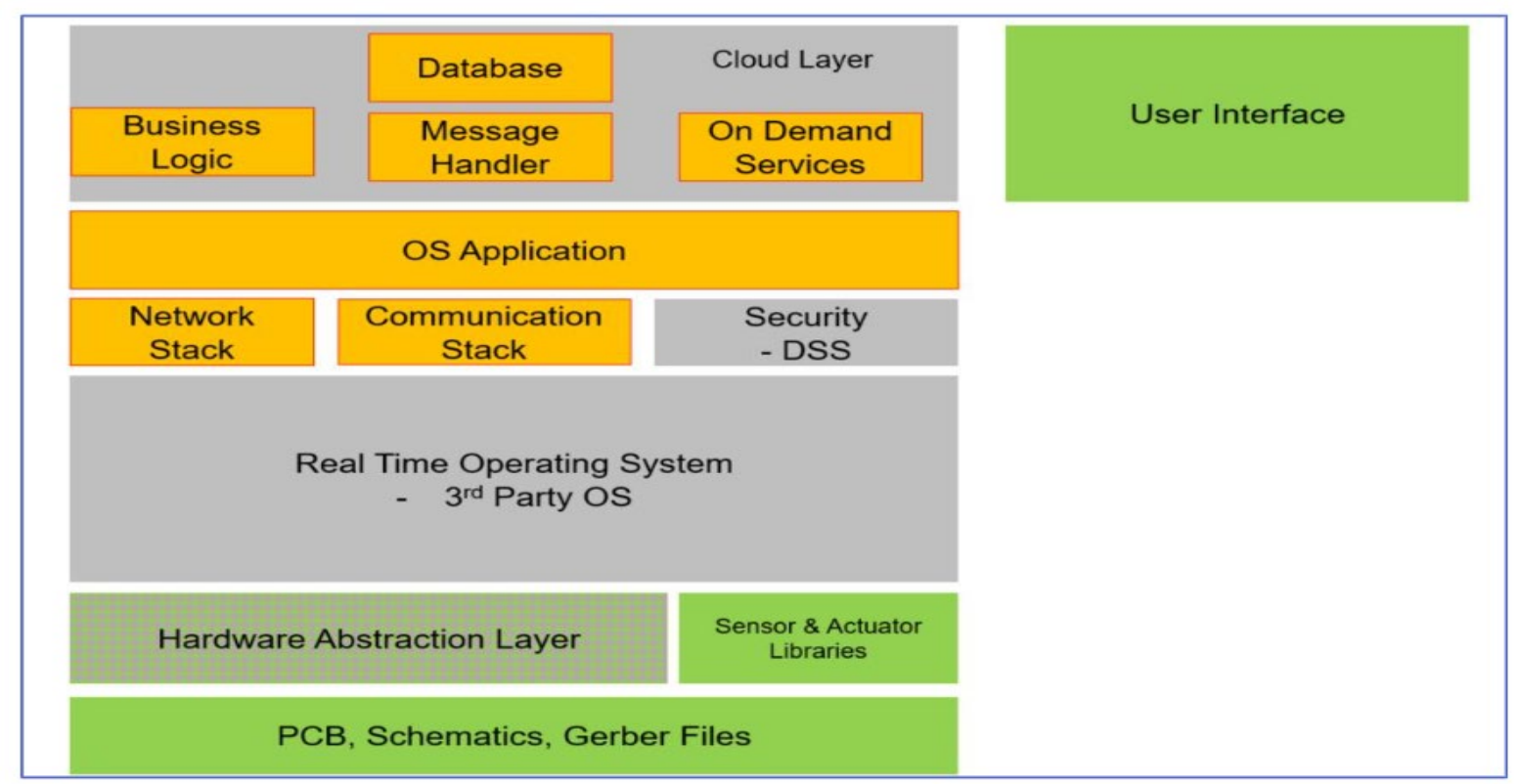

Fig.4.Smart Home Platform -Value Stream

Phase 2: Requirement Engineering (Analysis, Data / Control Flows)-The purpose of this project to develop a framework where on boarding of different smart components required in smart home system can be easily done. Hence, the main focus is the implementation of the following with an objective in mind that the system must allow boarding of any smart components relevant for smart home.

\section{-Sensor Hub-Smart Light: -Data or Control Flow}

There are three ways through which instructions can be issued:--Using Alexa Speaker-Using WEB GUI-Using Mobile Application5.1.1Data or Control for Smart Light Referring to control flow as shown in Figure 5: Smart Light Control Flow, when auto mode is chosen, the system monitors the light level. If the light level is low the system changes the light state to "on" when the light level is high the system changes the light state to "off". In manual mode, light state set by the user. If the light state is "on" it changes the state of the light to "ON". Whereas if the light state set by is "off", then light to "off" and it is shown in Figure 6.

Elaborating it further with respect to applicability, there are three ways through which an user interfaces with the hardware and those are using Alexa, Web GUI and Mobile Application. On AWS Implementation for smart Light, Alexa triggers lambda, which further triggers to IoT Core and from there, to hardware. While using Web GUI, action can be initialized to make changes in the smart light table, table will be created in dynamoDB and this change triggers AWS lambda, which further check the status of the topics and update the IoT topics which is subscribed by smart light hardware that has subscribed the topics. Data flow for smart light is depicted in Figure7.Information process in Figure8and system design components in Figure9. 
NOTE:-When auto mode is chosen, the system monitors the light evel. If the light level is low the system changes the light state to

"on" when the light level is high the system changes the light state to
"off".

When the system is in the manual mode, it checks the light State set by the user. If the light state set by the user is "on the state set by the user is "off" system changes the state of the light to "off"
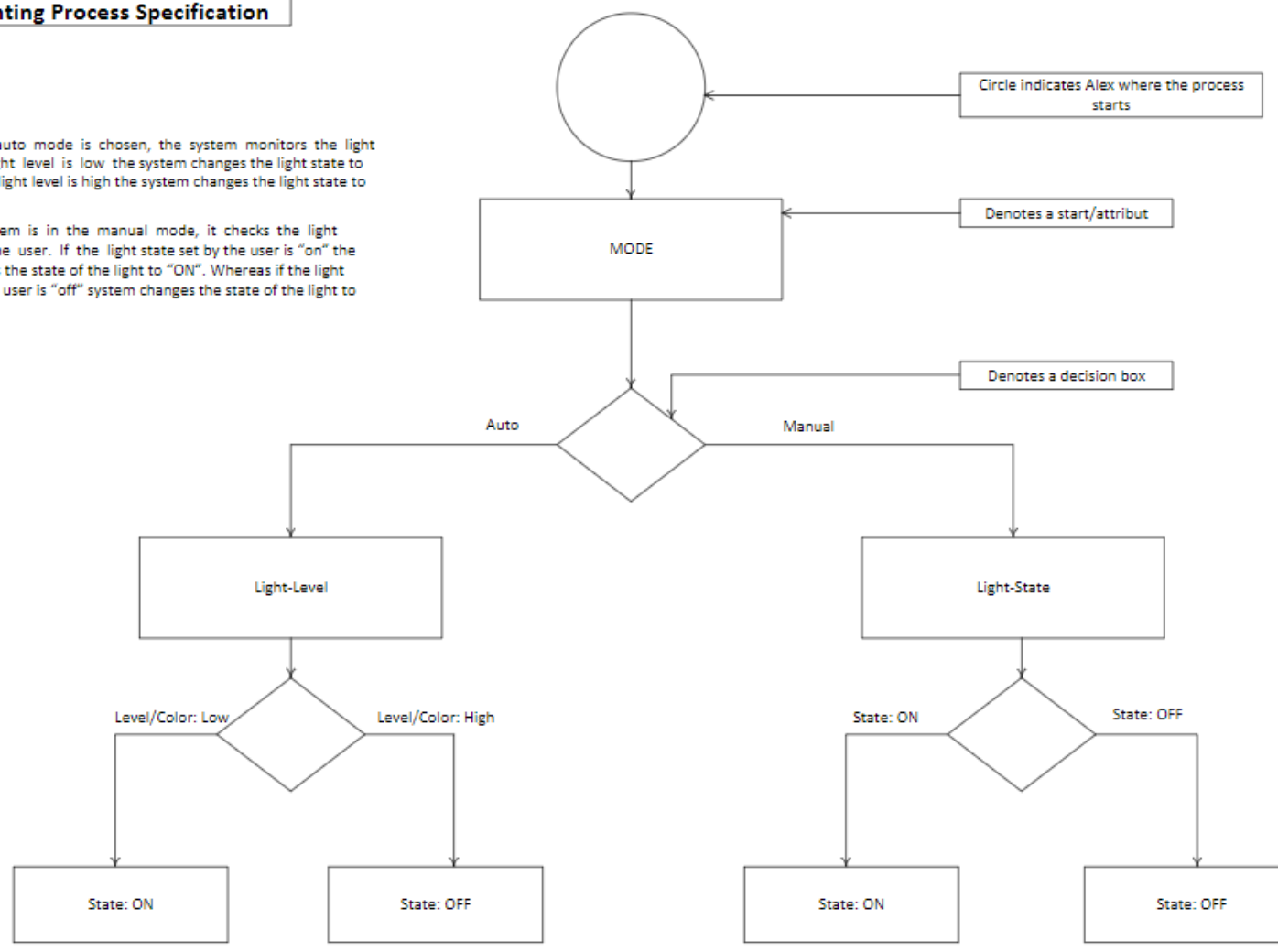

Fig. 5. Smart Light process specification
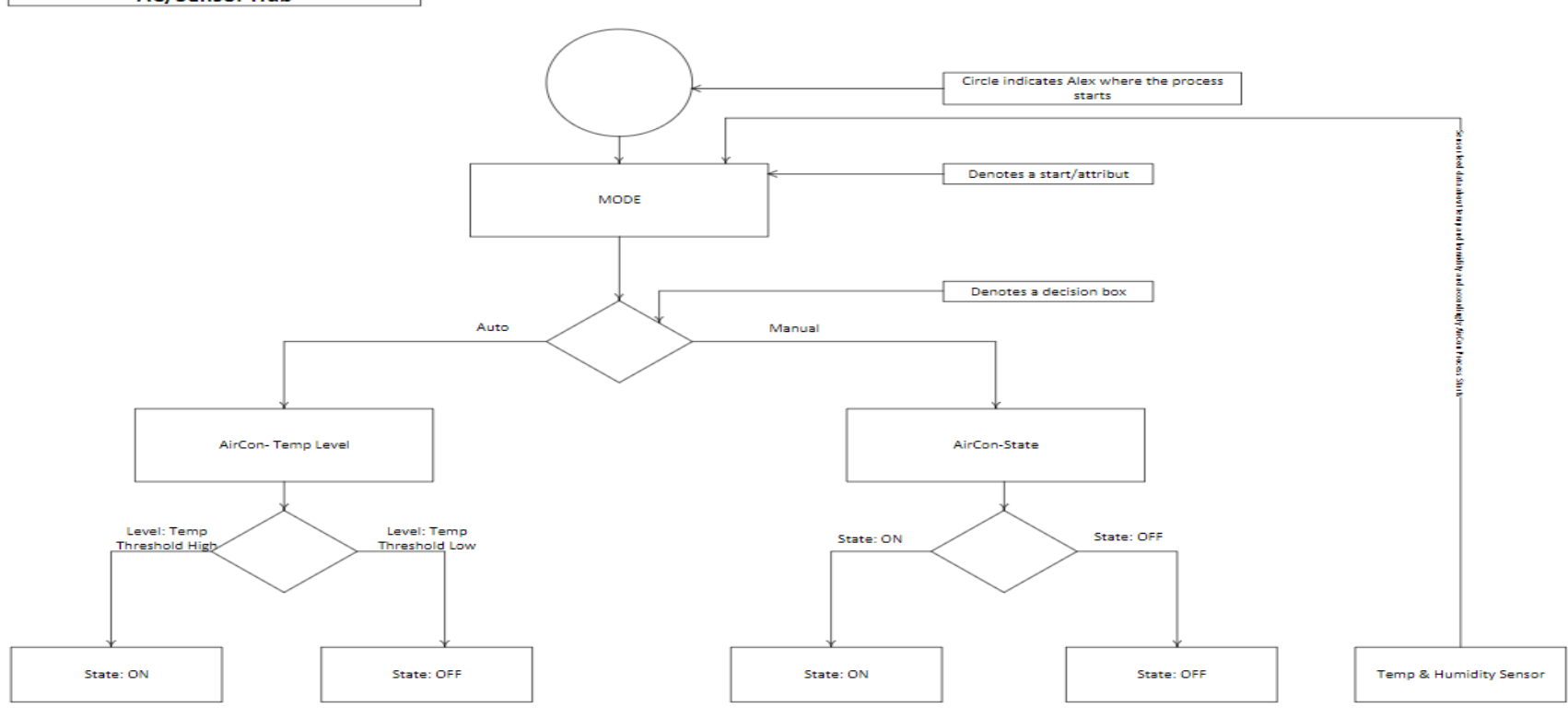

Fig.6. Smart Light Control Flow 


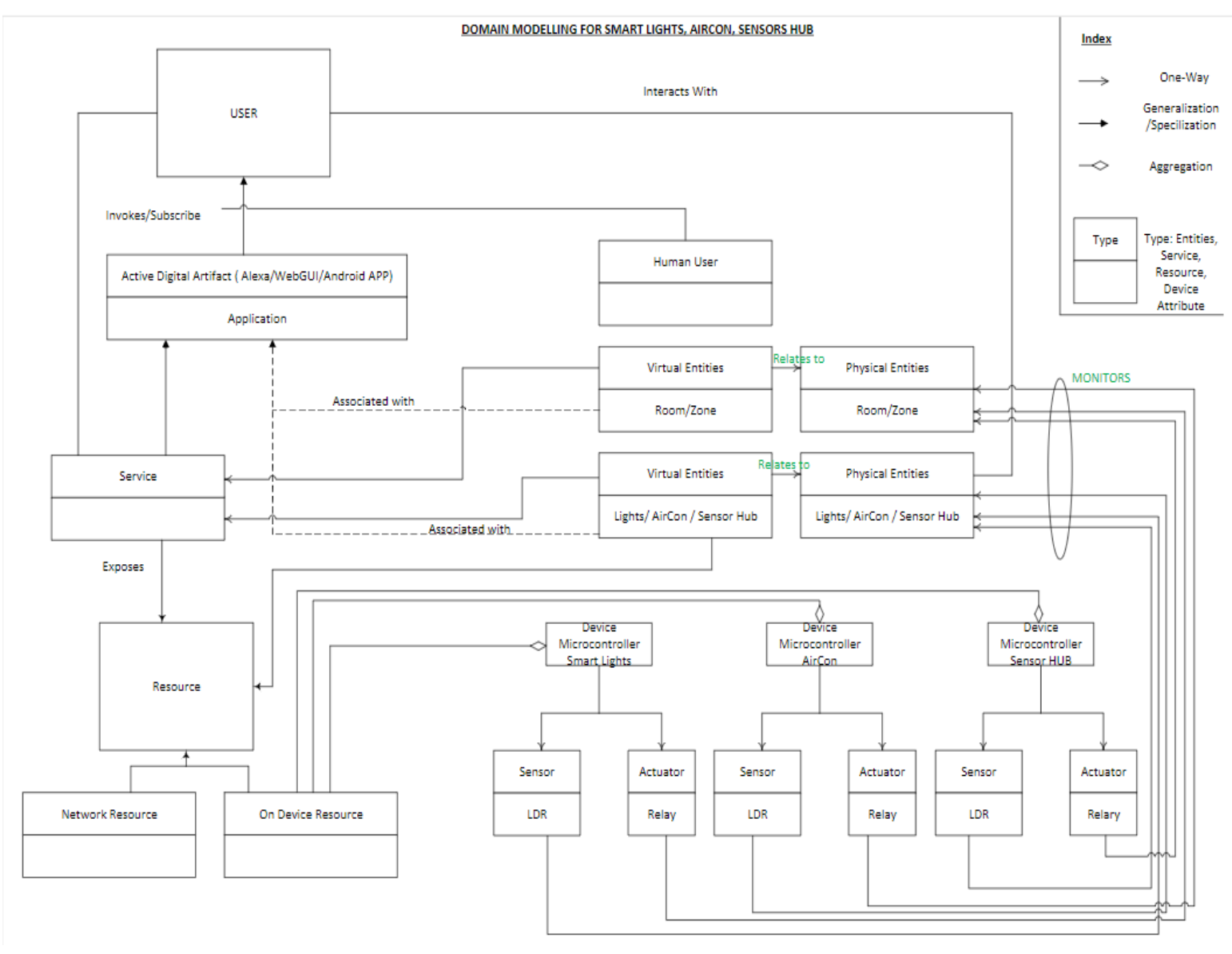

Fig.7.Data Flow for Smart Light

Information Process System

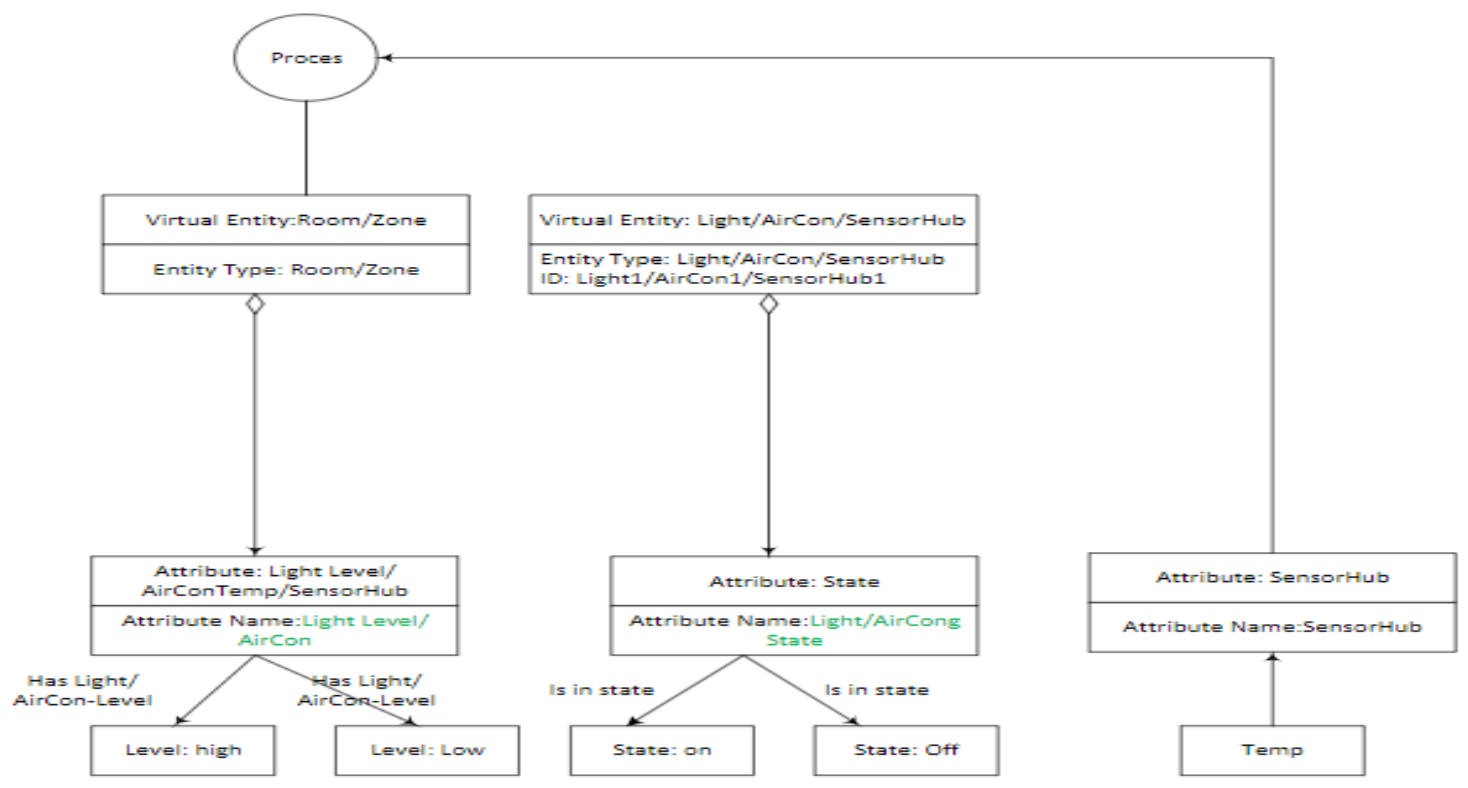

Fig.8.Information process system 

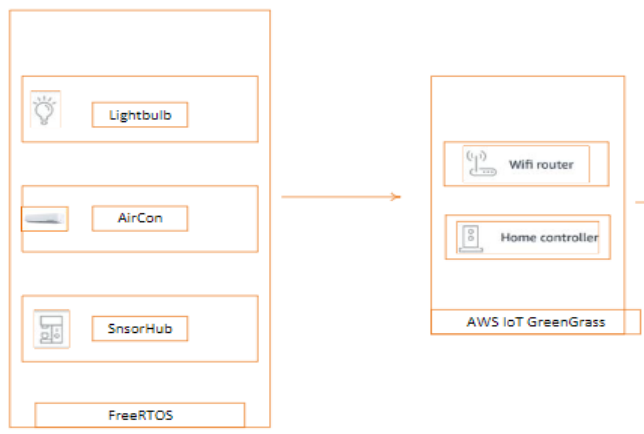

Fig.9.System Design Components

Hardware Requirement

Following are the hardware specifications which are used for this project-XMC4800:-main Controller-OPTIGA ${ }^{\mathrm{TM}}$ Trust X:-hardware security module-DPS310for simulating sensor hub:-The barometric pressure sensors DPS310 offers excellent pressure noise performance and high stability with temperature-RGB LED Lighting Shield with XMC1202:-for smart light. The Edge Block Diagram for Smart Light, Smart AC and Sensor Hub is depicted in Figure 10 and Figure 11.
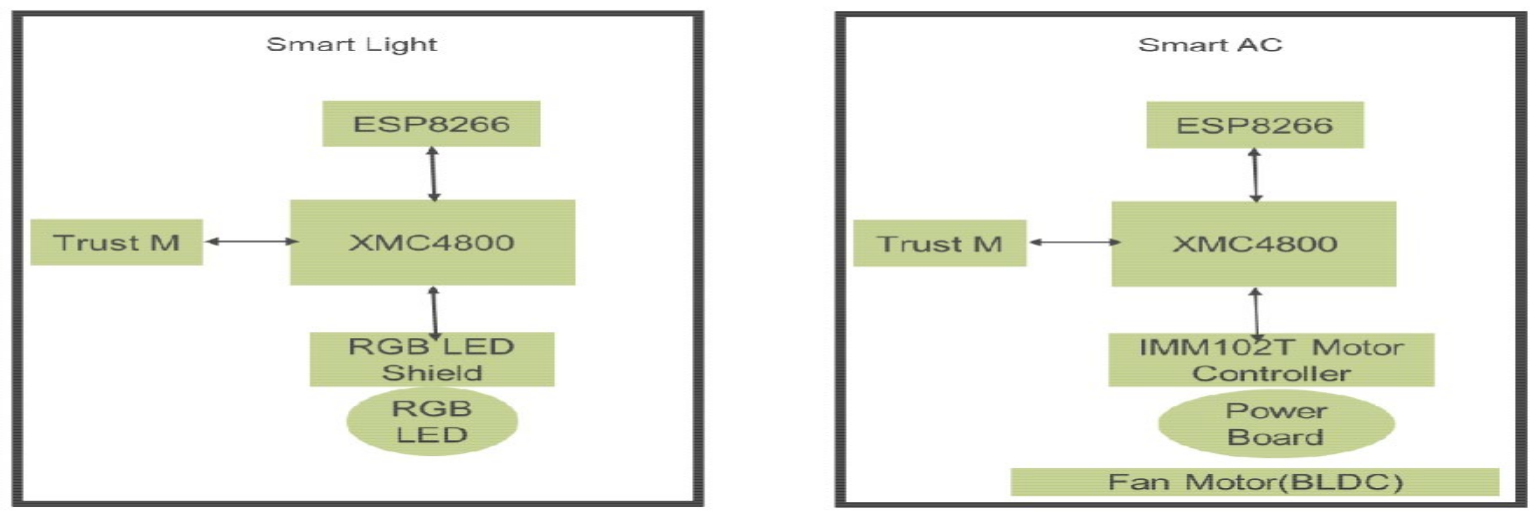

Fig.10. Edge Node Block Diagram -Smart AC and Smart Light

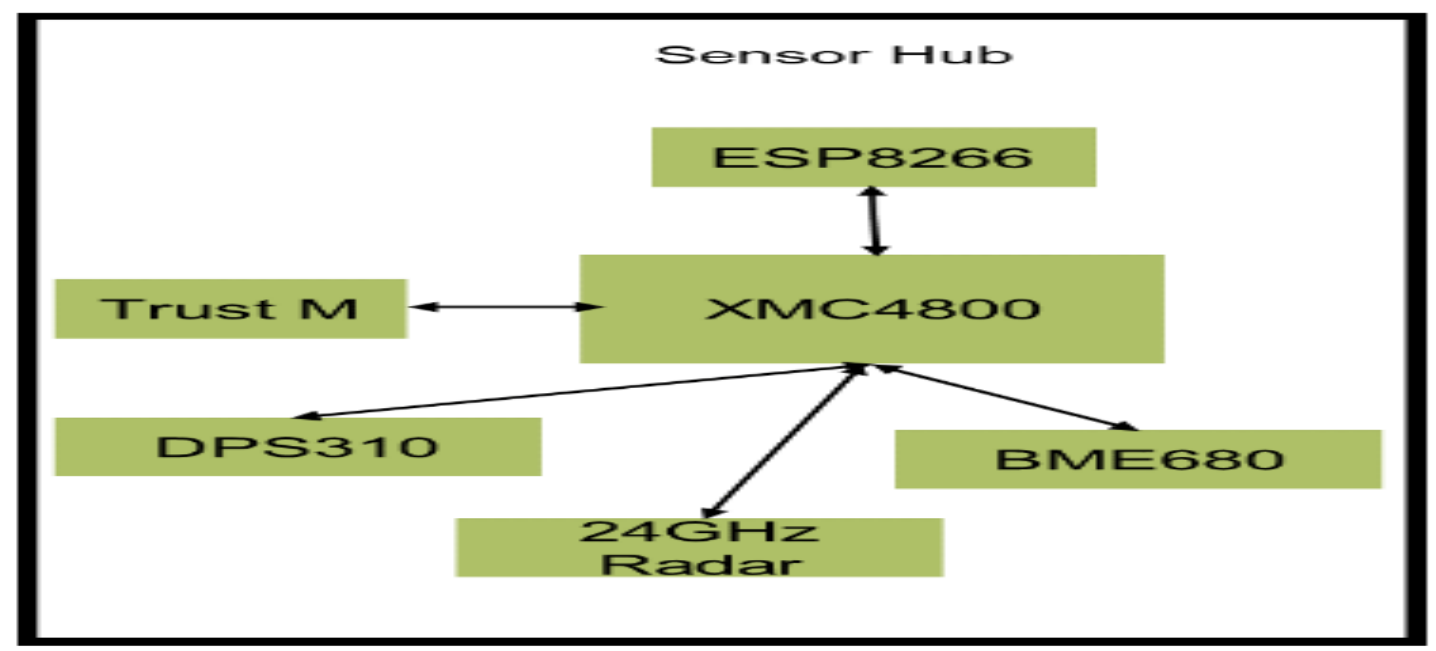

Fig.11.Edge Node Block Diagram-Sensor Hub 
Software RequirementFollowing are:

-Alexa Skill Kit-Mobile App-Web GUI-Dynamo -DB-AWS Lambda-AWS IoT

Following section of the document depicts the software interface with hardware:

- Figure 12shows the architecture breakdown for the hardware controlled by alexa.

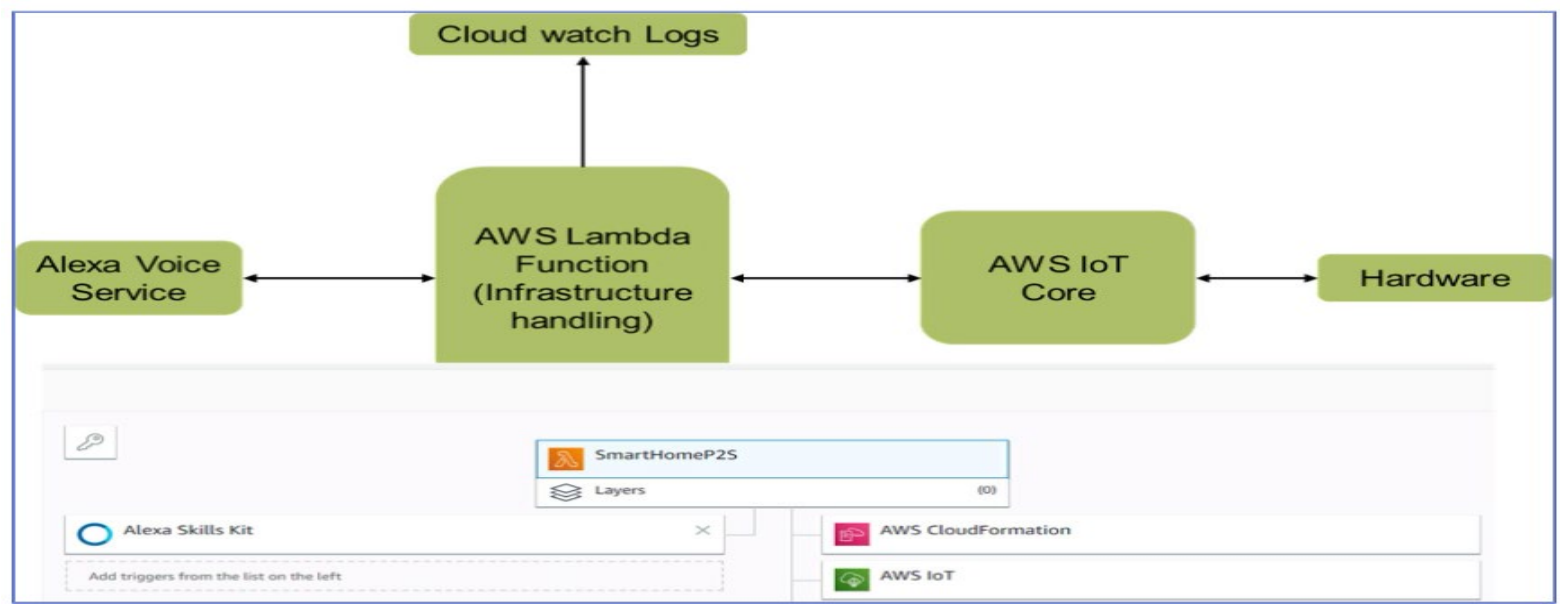

Fig.12.Architecture Breakdown: Alexa Controlled Hardware

Smart Light: It is one of the most important part of home automation and is equally simple in construction.

Refer Figure 13 for the process of controlling a smart light.

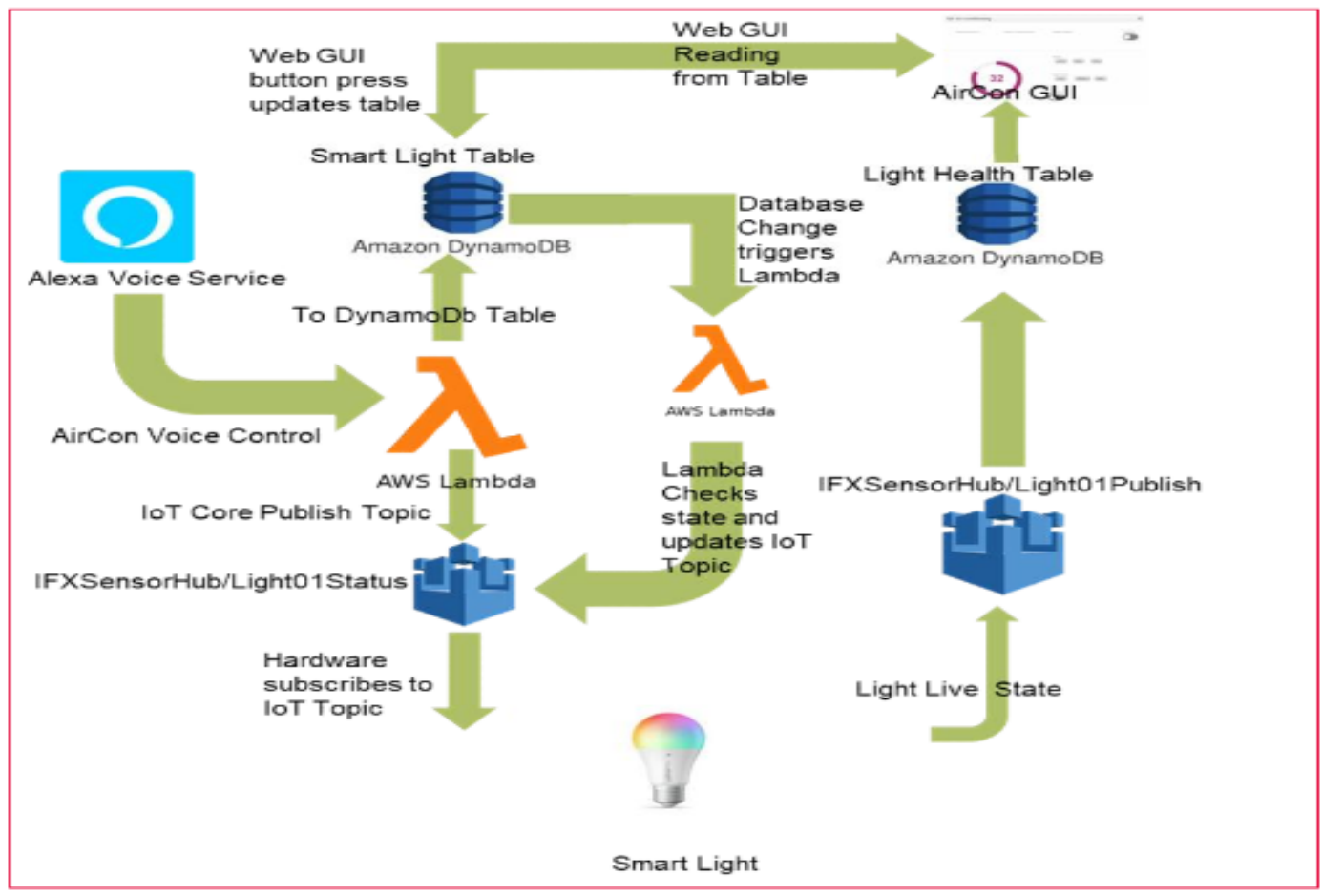

Fig.13. Controlling a smart light 
Smart AirConditioner:

Figure 14 for the process behind automation of an air conditioner. Though it looks complicated, it is pretty easy when its understood.

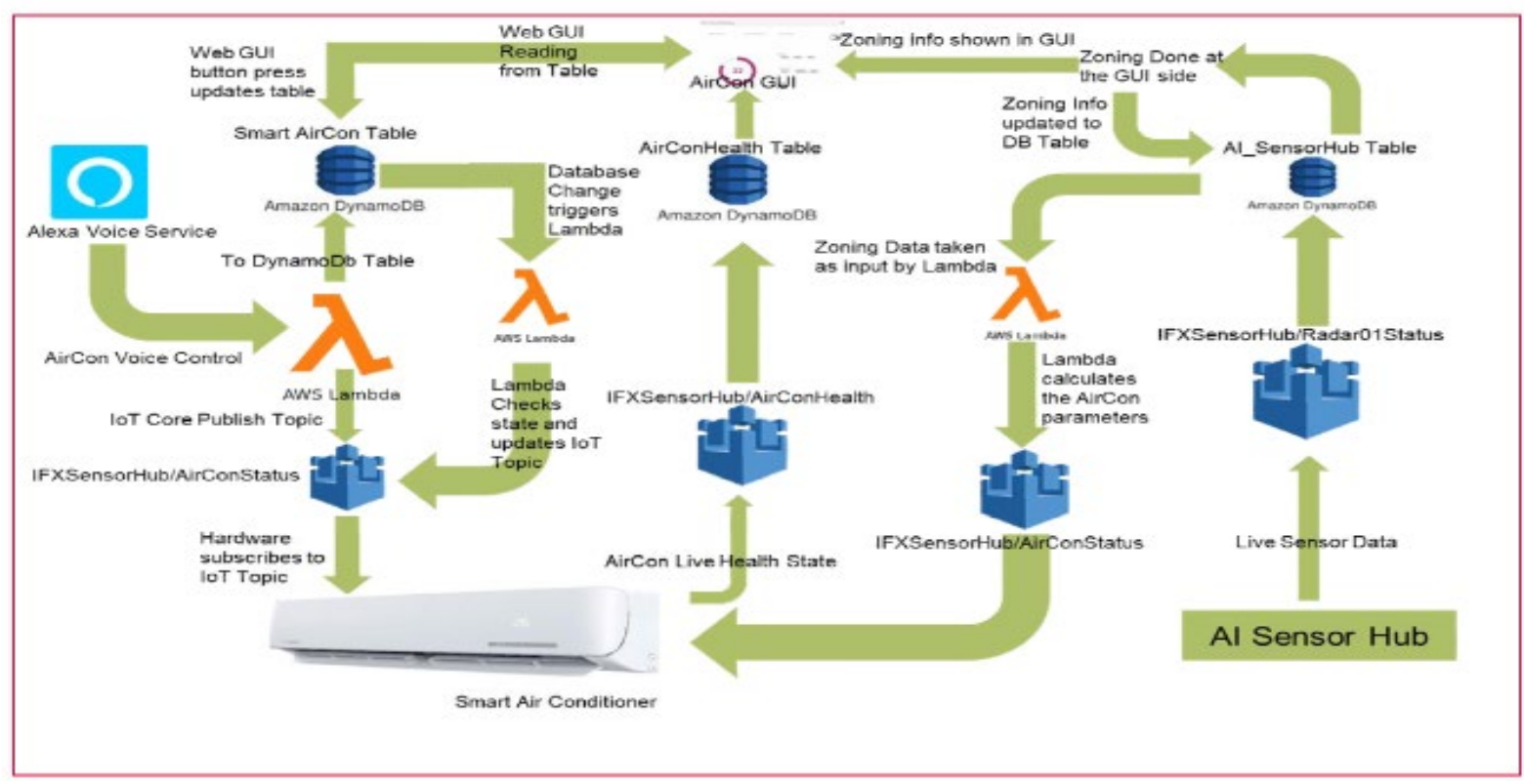

Fig.14.Controlling an air conditioner

The following pages contain theFigures 15-19 - architecture breakdown for different processes done before a light/ $\mathrm{A} / \mathrm{C}$ can be automated

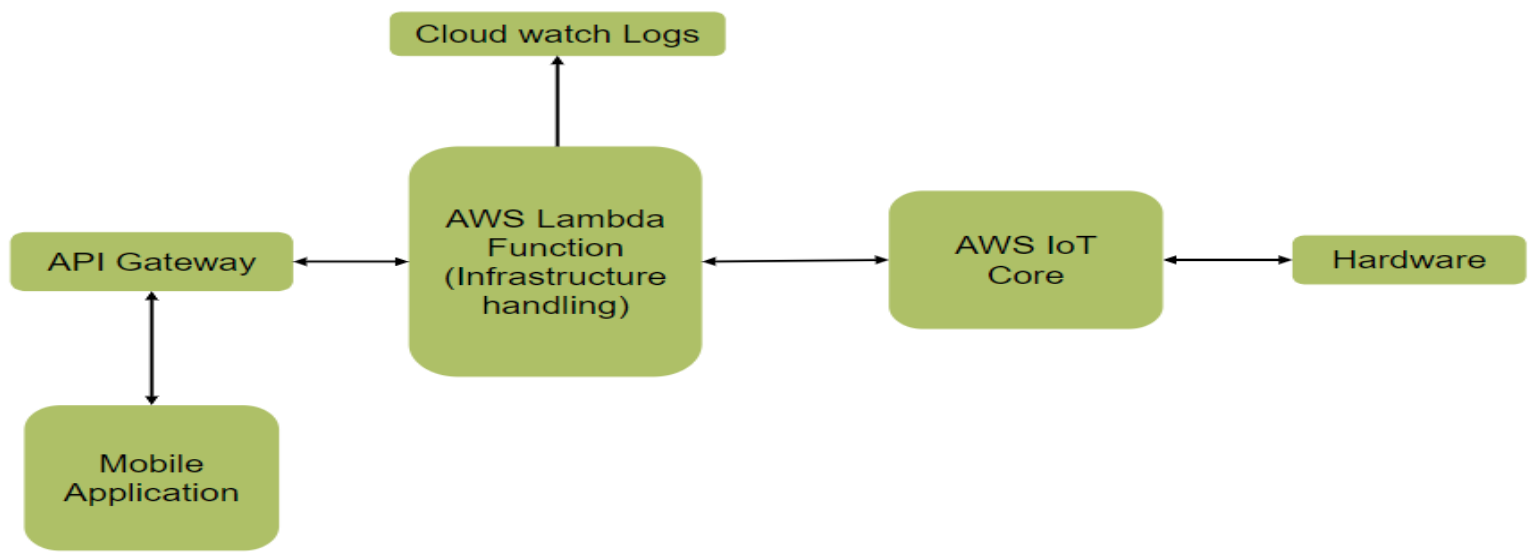

Fig.15.Architecture Breakdown: Android to Hardware 


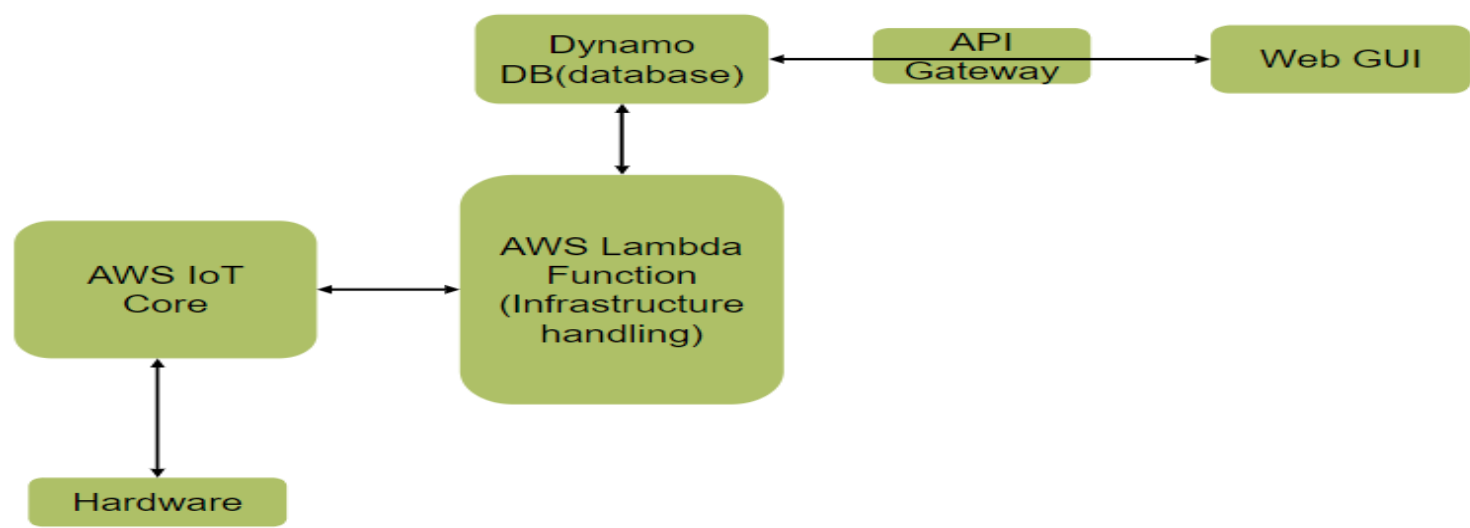

Fig.16. Architecture Breakdown: Web GUI to Hardware

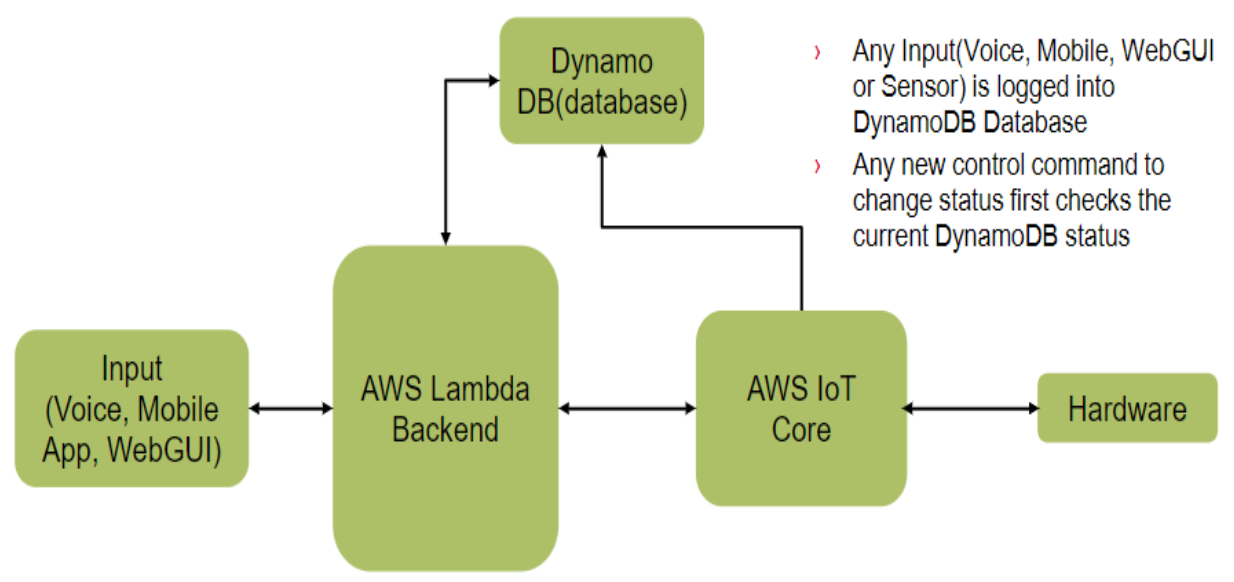

Fig.17.Architecture Breakdown: Data Storage

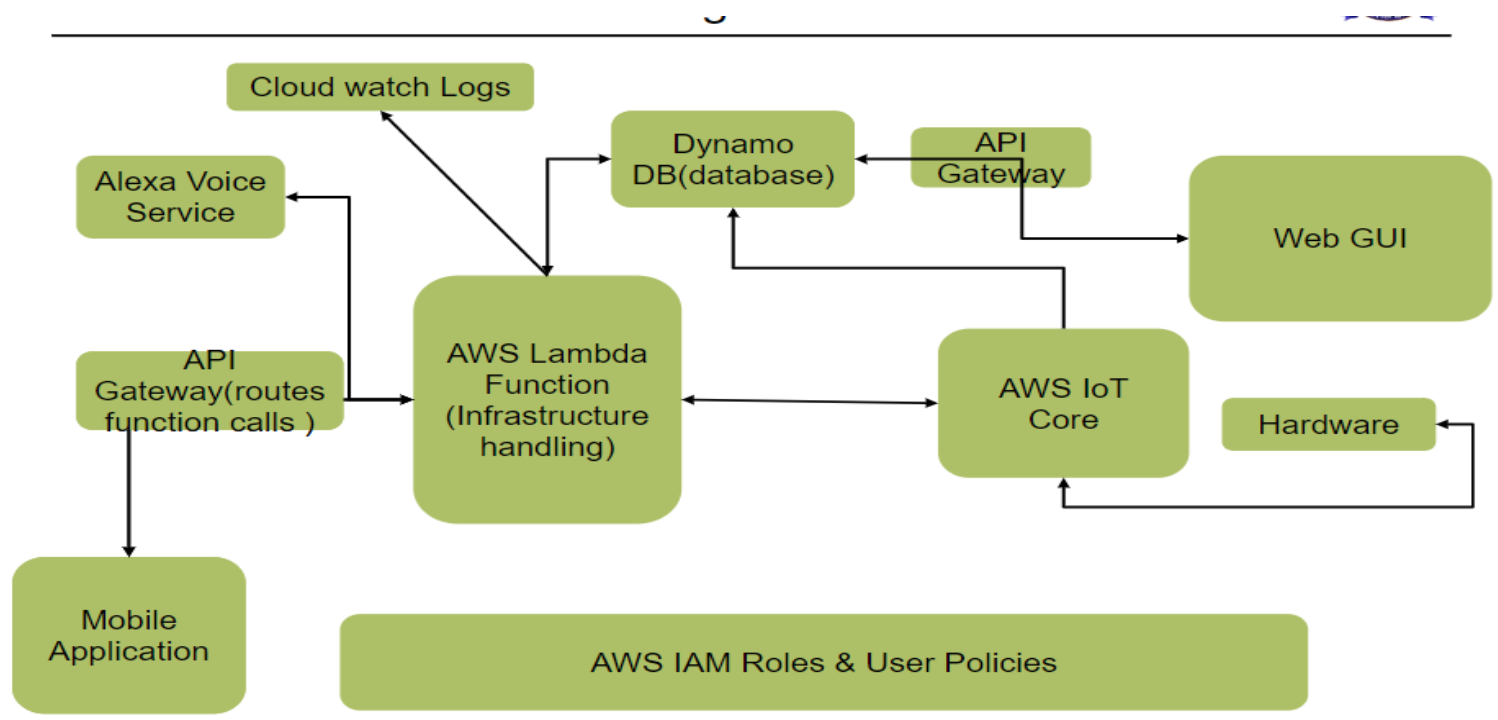

Fig.18.Architecture Breakdown :AWS Stack Integration 

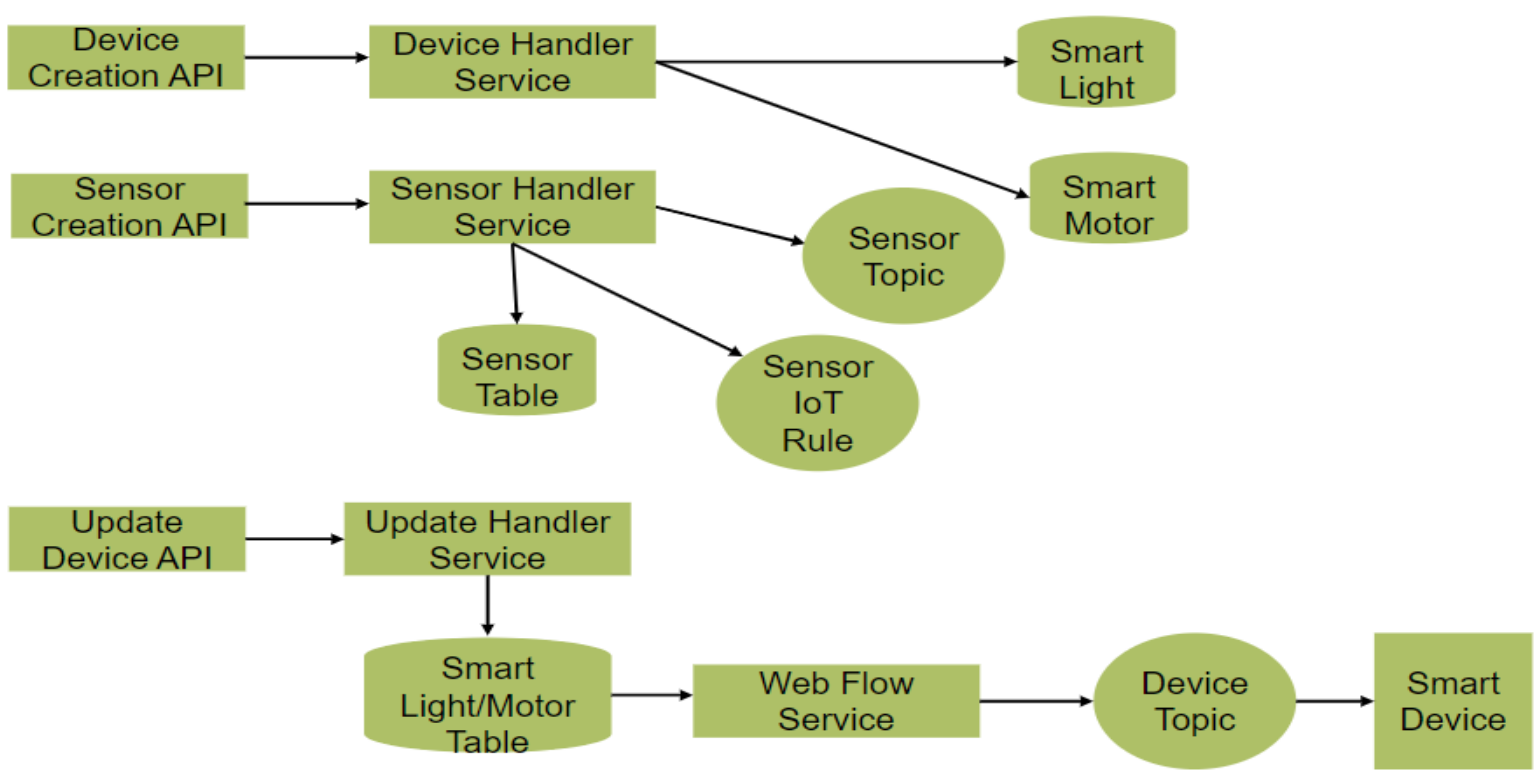

Fig.19. Architecture Breakdown :Data flow for Device Creation, Sensor Creation and Sensor Update

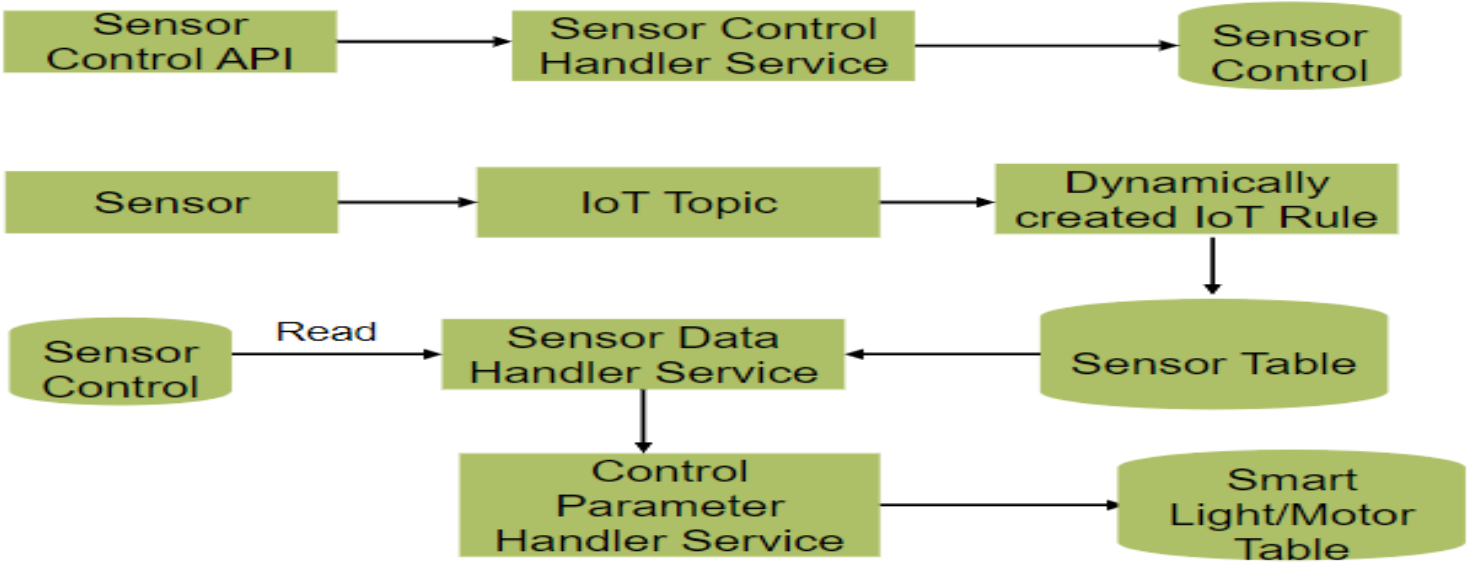

Fig.20.Architecture Breakdown: Sensor Control Flow

The following contains the code and its use. See Figures 20 - 36

Endpoint/create_device

Creates Smart

Device of type

Light or Motor or Aircon

Sample Payload

$\{$ "uniqueName":"diningRoomLight", "deviceType":"Light" \}

Fig. 21.Code for- Creatinga Device 
Sample Payload

\{ $\quad$ "sensorName":"temperature1"\}

Fig.22.Code for- Creating a Sensor

Endpoint/update_light_para meter

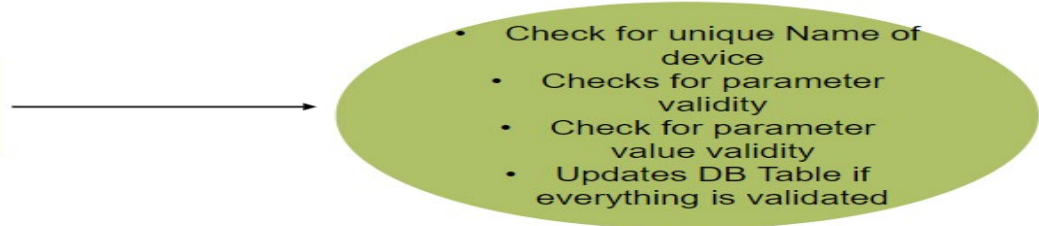

Sample Payload

f "uniqueName":"bedroom 1",

"parameter":"colour",

"value":"red" \}

Fig.23.Code for- Update Light

Endpoint/update_motor_par ameter
Check for unique Name of device

- Checks for parameter

validity

- Check for parameter value validity

- Updates DB Table if everything is validated

Sample Payload

$\{$ "uniqueName":"motor2",

"parameter":"mode",

"value":"cool"“ \}

Fig.24.Code for- Update Motor 
"application":"SmartMotor",

"application":"Smartmotor", 1 "controlledDevice":"bedRoom1",

"controlledDevi

"conditionalExpression":"Greater",

"controlledValue":" 1 ",

"controlledParam":"status"

"controlparam":"temperature".

\}.

"conditionalvalue":"35"

"ensorName":"temperature1"

\section{Fig.25.Code for- Sensor Control}

-The following Figures 25-28 shows various device interactions.

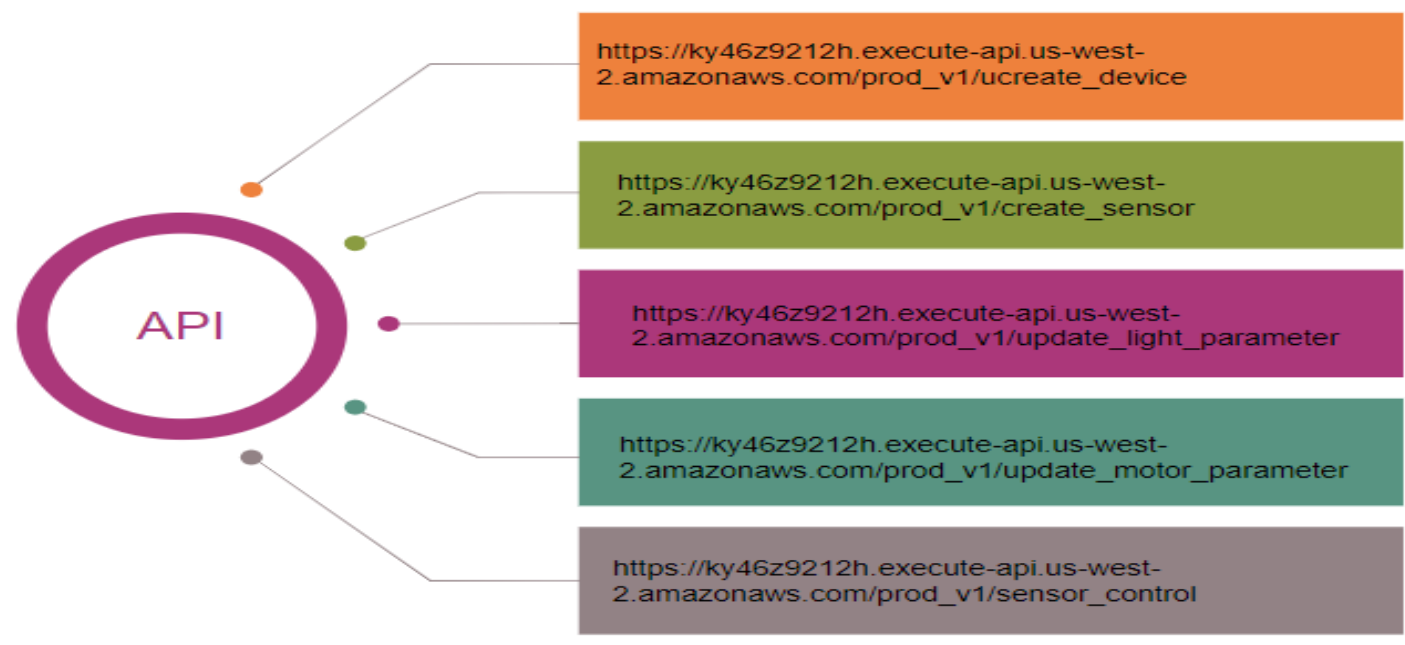

Fig.26.Inter Device Interaction 


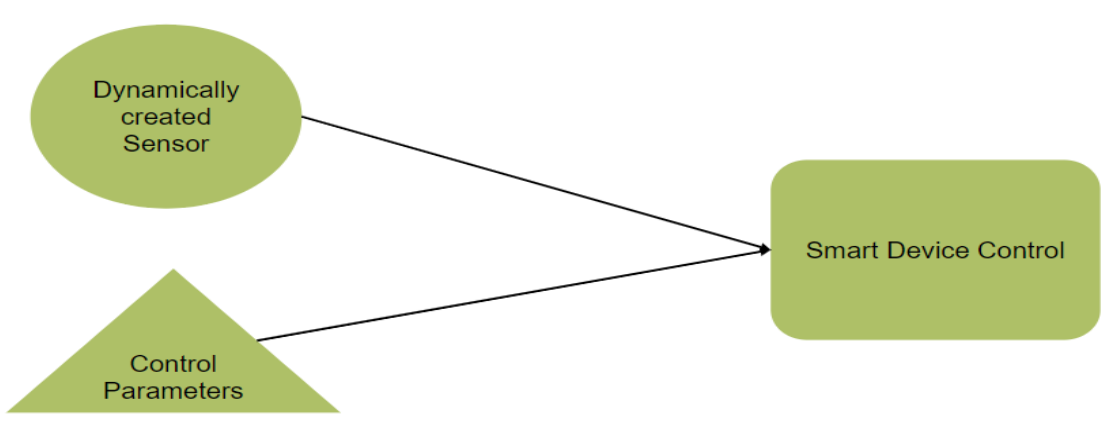

Sensor created at runtime and given control parameters at random control end actuation devices - Light or Moto

Fig.27.Inter Device Interaction

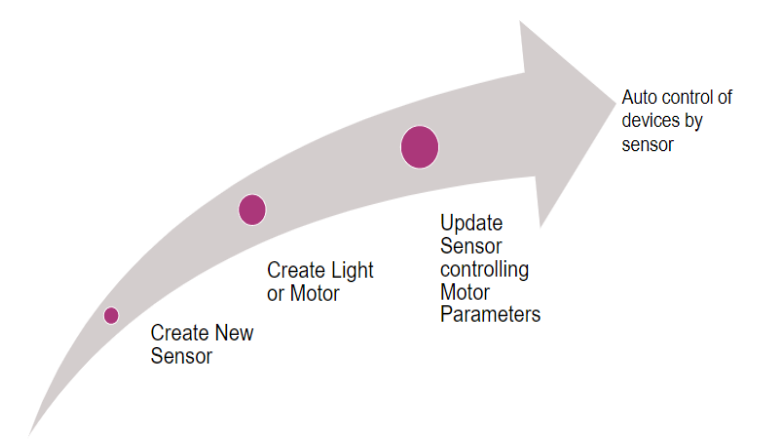

Fig.28.Inter Device Interaction
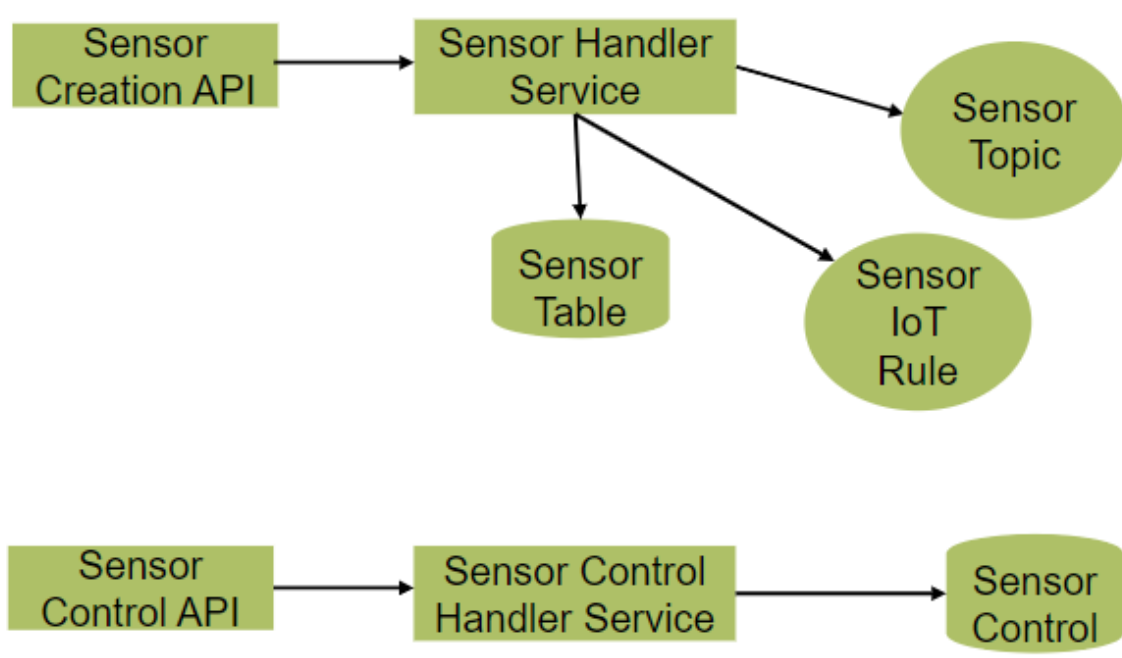

Fig.29. Device Interaction

The following Figures 29-30 shows the architecture and data flow as a general case and for smart light 


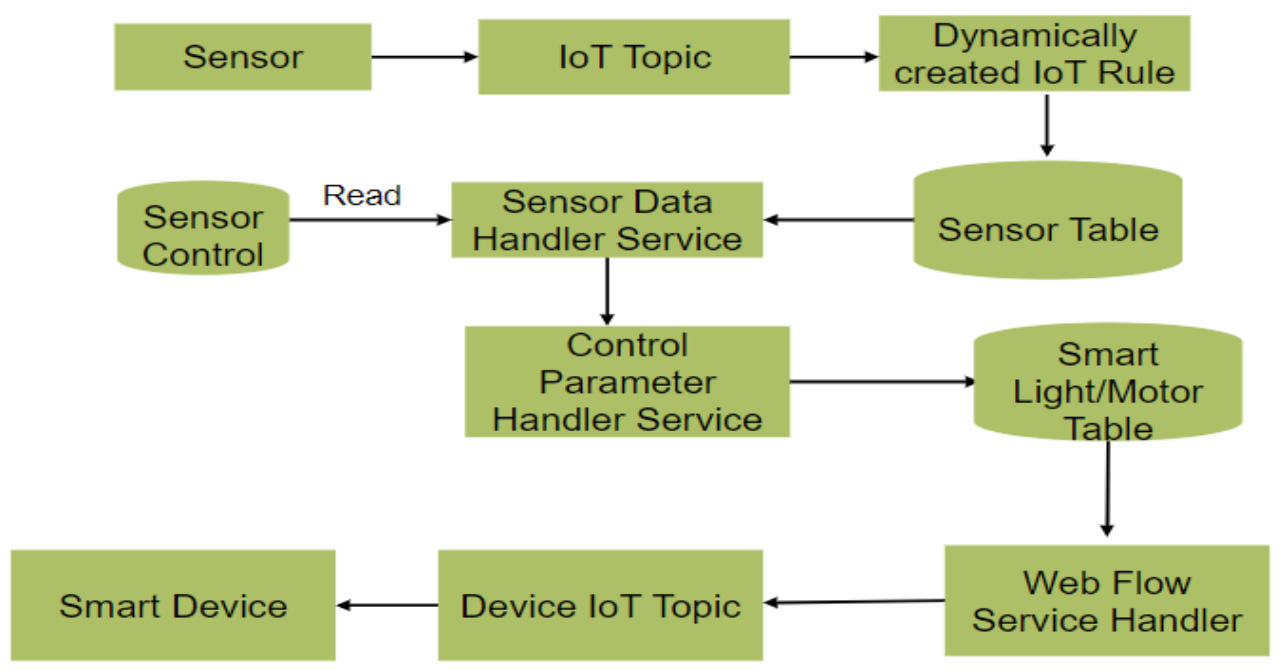

Fig.30.Architecture \& Data Flow : Creation

\{

"brightness": "255",

"colour": "grey",

"mood": "null",

"origin": "web",

Smart Light Data

Model

"status": "1",

"uniqueName": "bedroom"

\}
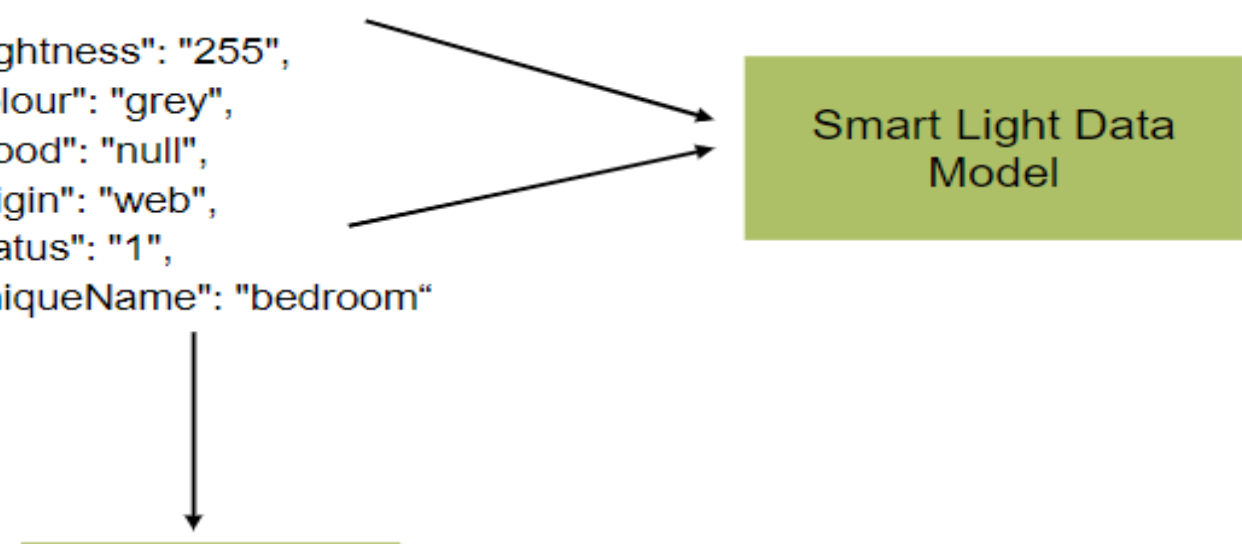

Primary Key

Fig.31.Architecture \& Data Flow : Auto Control ; Smart Light 
Table 1. Smart light : data modelling details

\begin{tabular}{|c|c|c|c|c|c|}
\hline Item & Meaning & $\begin{array}{l}\text { Data } \\
\text { Type }\end{array}$ & $\begin{array}{l}\text { Typical } \\
\text { Value }\end{array}$ & $\begin{array}{l}\text { Comprehensive List of } \\
\text { Value/Range }\end{array}$ & Comments \\
\hline uniqueName & $\begin{array}{l}\text { Unique ID of } \\
\text { Smart Light }\end{array}$ & String & bedRoom & $=$ & Primary Key \\
\hline origin & $\begin{array}{l}\text { Denotes the } \\
\text { origin of the } \\
\text { database } \\
\text { update }\end{array}$ & String & web & alexa/web/radar & $\begin{array}{l}\text { This column is used to } \\
\text { differentitate between } \\
\text { different points of origin of } \\
\text { the database update. }\end{array}$ \\
\hline brightness & $\begin{array}{l}\text { Brightness of } \\
\text { Light }\end{array}$ & String & 24 & $0-255$ & \\
\hline colour & Light Colour & String & red & $\begin{array}{l}\text { Red/green/black/yellow/ } \\
\text { marron etc }\end{array}$ & Default - Red \\
\hline $\operatorname{mood}$ & Mood Lighting & String & reading & Reading/sleep/study etc & Default - Study \\
\hline status & $\begin{array}{l}\text { Current on/off } \\
\text { status }\end{array}$ & String & 0 & 0,1 & $\begin{array}{l}\text { On calling this function by } \\
\text { User, aircon is turned on } \\
\text { with default mode,no } \\
\text { swing and default } \\
\text { temperature of " } 24 \text { ". }\end{array}$ \\
\hline
\end{tabular}

The following Figures 31-32 shows the architecture and data flow for a smart motor $(\mathrm{A} / \mathrm{C})$ -

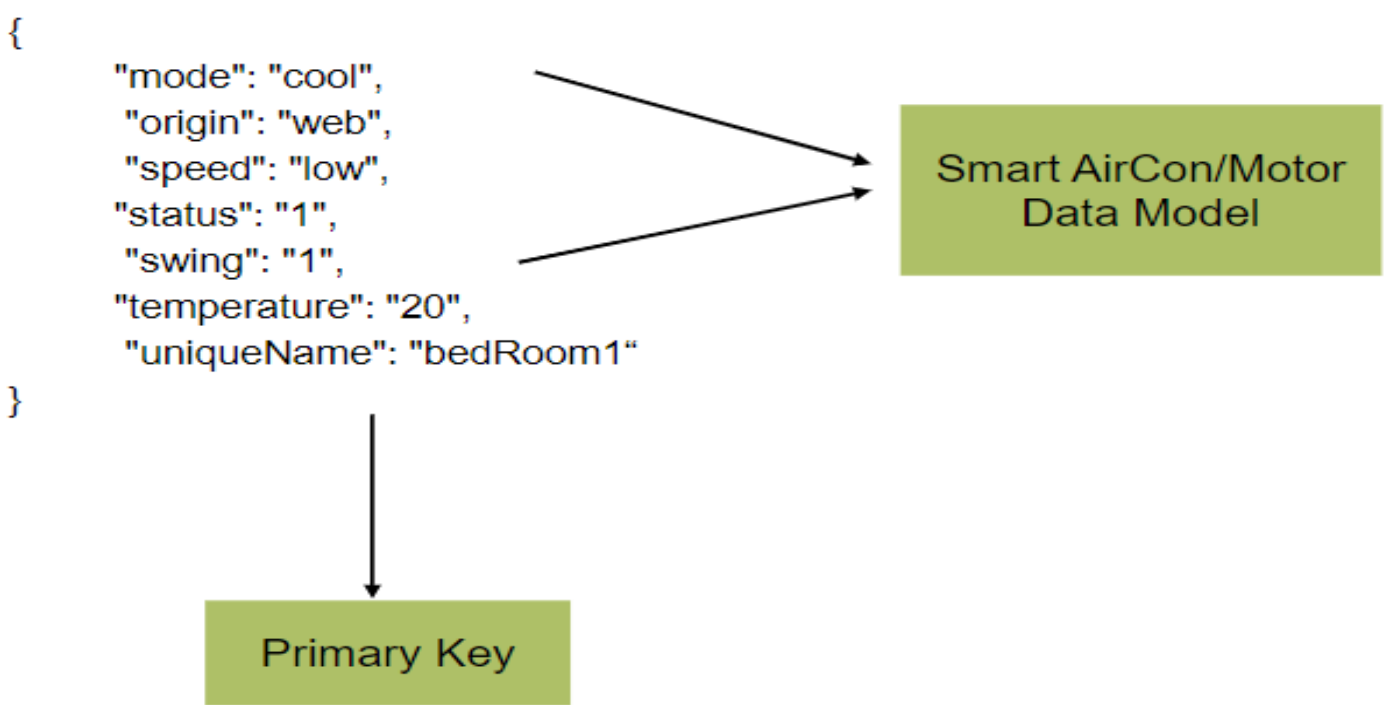

Fig.32. Architecture \& Data Flow : Auto Control ; smart A/C (motor) 


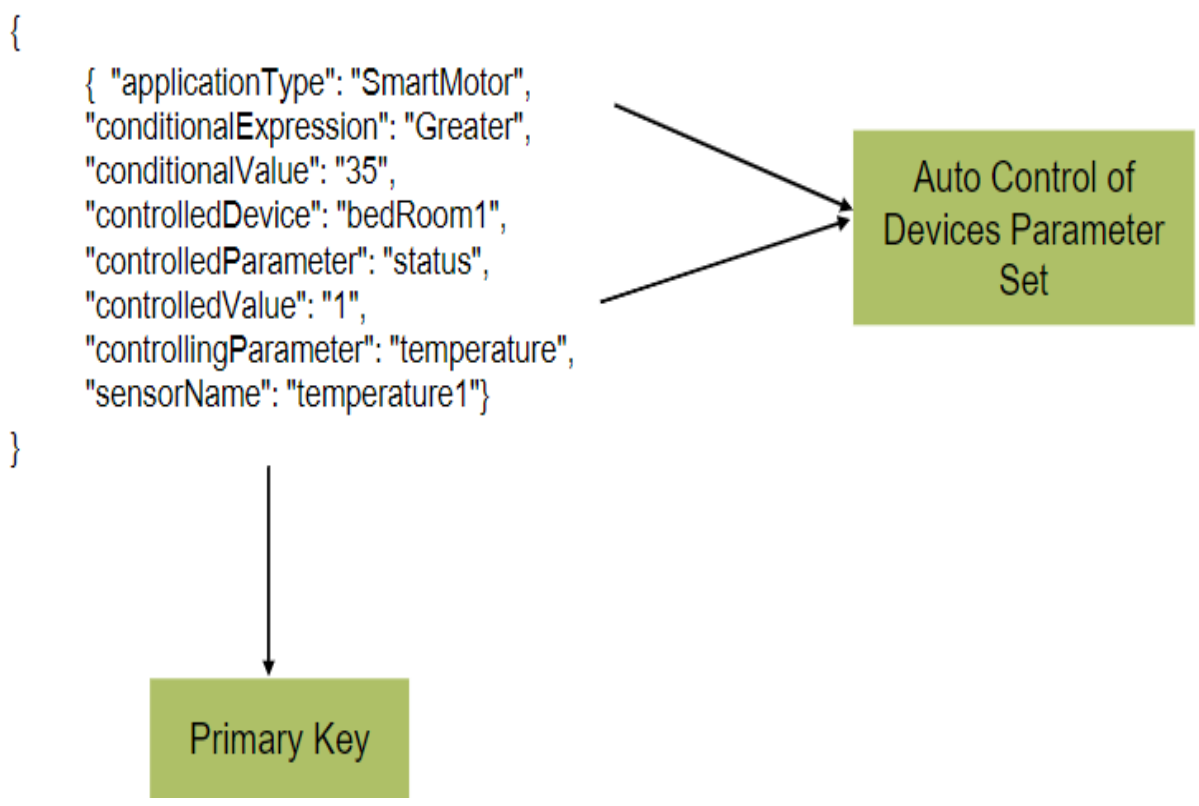

Fig.33.Architecture \& Data Flow : Auto Control ; smart A/C (motor)

Table 2. Smart Motor : Data Modelling Details

\begin{tabular}{|c|c|c|c|c|c|}
\hline Item & Meaning & $\begin{array}{l}\text { Data } \\
\text { Type }\end{array}$ & $\begin{array}{l}\text { Typical } \\
\text { Value }\end{array}$ & $\begin{array}{l}\text { Comprehensive List of } \\
\text { Value/Range }\end{array}$ & Comments \\
\hline uniqueName & $\begin{array}{l}\text { Unique ID of } \\
\text { Smart Motor }\end{array}$ & String & bedRoom & * & Primary Key \\
\hline origin & $\begin{array}{l}\text { Denotes the } \\
\text { origin of the } \\
\text { database } \\
\text { update }\end{array}$ & String & web & alexa/web/radar & $\begin{array}{l}\text { This column is used to } \\
\text { differentitate between } \\
\text { different points of origin of } \\
\text { the database update. }\end{array}$ \\
\hline temperature & $\begin{array}{l}\text { Temperature of } \\
\text { the AirCon Unit }\end{array}$ & String & 24 & $17-31$ & 24 \\
\hline mode & AirCon Modes & String & auto & Cool/auto/fan & auto \\
\hline fanSpeed & $\begin{array}{l}\text { Speed of the } \\
\text { Aircon fan }\end{array}$ & String & medium & High/medium/low/auto & auto \\
\hline swing & $\begin{array}{l}\text { Swing of AirCon } \\
\text { Flap }\end{array}$ & String & 0 & 0,1 & Default - 0 \\
\hline status & $\begin{array}{l}\text { Current on/off } \\
\text { status }\end{array}$ & String & 0 & 0,1 & $\begin{array}{l}\text { On calling this function by } \\
\text { User, aircon is turned on } \\
\text { with default mode, no swing } \\
\text { and default temperature of } \\
\text { " } 24 \text { ". }\end{array}$ \\
\hline
\end{tabular}


Table 3. Auto Control of motor: Data Modelling Details

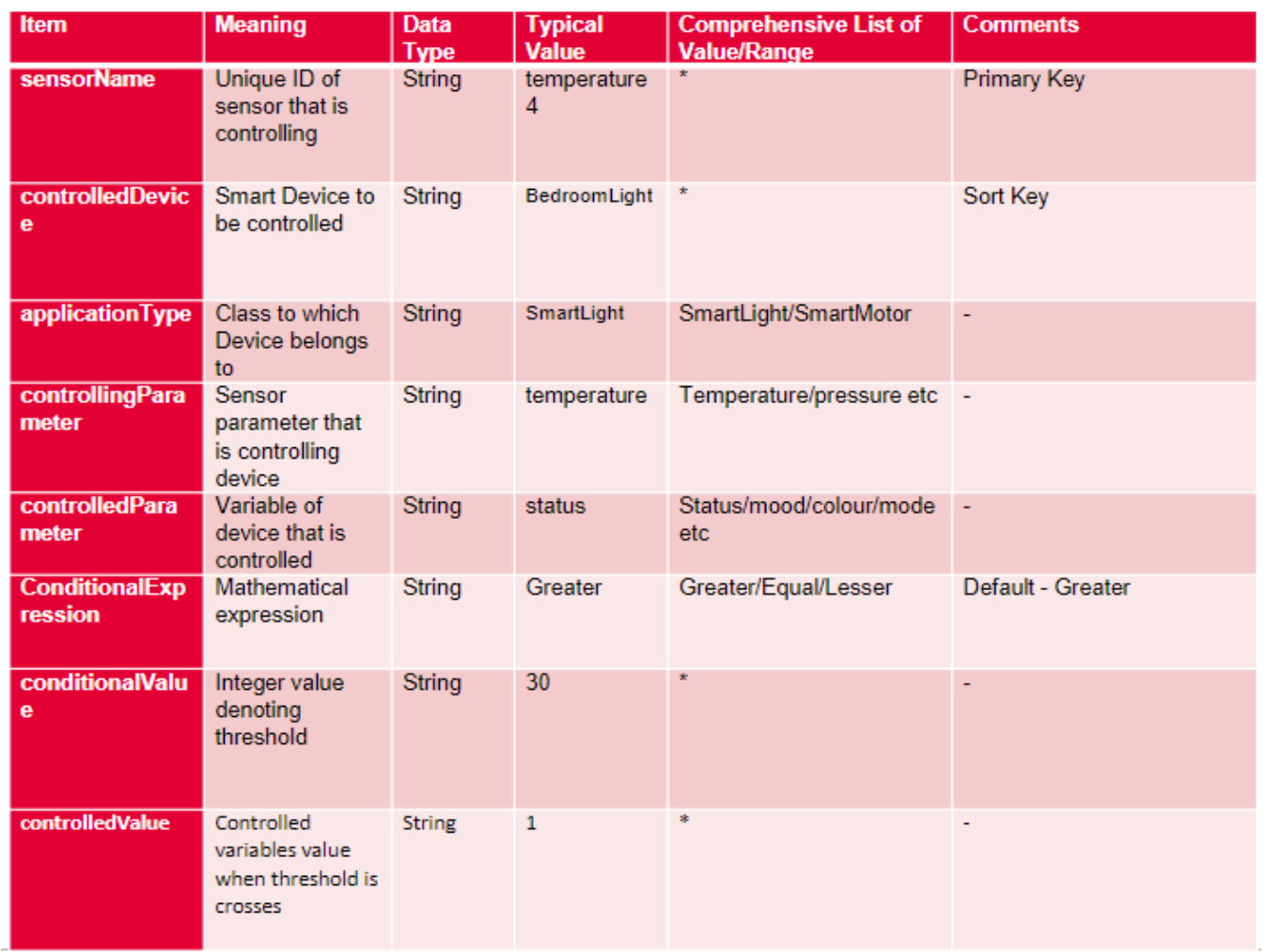

\section{Conclusion}

Cloud platforms for IoT play a vital role for Smart Cities. This paper elaborates a scalable cloud model for Internet of Things based on the concept of modularization and service integration the cloud model can be implemented on a small scale in the initial phase, and be extended and integrated in the future. Smart Home in a scaled down manner highlighting interconnection between different smart devices Seamless data transfer enhancing the user experience can be achieved easily and can be made viable due to its low cost. The design of our scalable cloud model can successfully response the challenges of IoT in Smart Home. The proposed architecture and implementation model provide a feasible solution to build a cloud platform for IoT, which supports the step-by-step construction of Smart Home.

\section{Reference}

[1] Hassan H., Jamaluddin R.A., Marafa F.M. (2019), Internet of Thing (IoT) Smart Home Systems: Conceptual Ethical Framework for Malaysian Developers. In: Badioze Zaman H. et al. (eds) Advances in Visual Informatics. IVIC 2019. Lecture Notes in Computer Science, vol 11870. Springer, Cham. https://doi.org/10.1007/978-3-030-34032-2_40

[2] Amazon Echo Homepage. 3https://slate.com/technology/2018/11/amazon-echo-alexa-murder-case-new-hampshirewarrant.html. Accessed 30 July 2019

[3] Al-Kuwari M, Ramadan A, Ismael Y, Al-Sughair L, Gastli A, Benammar M. Smart-Home Automation Using IoT-Based Sensing and Monitoring Platform, IEEE. 2018

[4] Saeed F, Paul A, Rehman A, Hong WH, Seo H. IoT-based intelligent modeling of smart home environment for fire prevention and safety. Journal of Sensor and Actuator Networks. 2018;7(1):11. DOI: 10.3390/jsan7010011

[5] Khan NS, Ghani S, Haider S. Real-time analysis of a sensor's data for automated decision making in an IoT-based smart home. Sensors. 2018;18:1711. DOI: 10.3390/s18061711

[6] Botta A, de Donato W, Persico V, Pescapé A. Integration of cloud computing and internet of things: A survey. Future Generation Computer Systems. 2016;56:684-700. 
[7]K. Vijayakumar, Chokkalingam Arun, "Integrated cloud-based risk assessment model for continuous integration”,Int. J. Reasoning-based Intelligent Systems”, Vol. 10, Nos. 3/4, 2018.

[8]K. Vijayakumar, S. Suchitra and P. Swathi Shri, "A secured cloud storage auditing with empirical outsourcing of key updates", Int. J. Reasoning-based Intelligent Systems, Vol. 11, No. 2, 2019. 